\title{
Microalgas: potencial para la producción de compuestos bioactivos nanoencapsulados
}

\author{
Microalgas: potencial para a produção de compostos bioativos nanoencapsulados
}

\section{Luis Guillermo Ramírez-Mérida ${ }^{1,2}$, Cristiano Ragagnin de Menezes², Leila Queiroz Zepka², Eduardo Jacob-Lopes ${ }^{2 *}$}

\author{
${ }^{1}$ Centro de Biotecnología Aplicada, Departamento de Biología, \\ Universidad de Carabobo - UC, Valencia, Venezuela \\ lgrm@gmail.com \\ ${ }^{2}$ Departamento de Tecnologia e Ciência de Alimentos, \\ Universidade Federal de Santa Maria - UFSM, Santa Maria, RS, Brasil \\ cristianorm@gmail.com; lqz@pq.cnpq.br; jacoblopes@pq.cnpq.br
}

\begin{abstract}
Resumen
Las microalgas se presentan como una fuente potencial de compuestos bioactivos para ser utilizado en la industria alimentaria generando alimentos funcionales. El desarrollo y búsqueda de alimentos funcionales, como método para mejorar y mantener la salud representa un campo de la investigación muy explorado en años recientes. Lípidos, proteinas, polisacáridos, compuestos fenólicos, carotenoides, entre otros bioproductos proveniente de microalgas pueden ser utilizados como fuente de ingredientes para productos funcionales, sin embargo, la inestabilidad química de muchos de ellos puede comprometer su acción. La nanoencapsulación representa una herramienta nanotecnológica capaz de proporcionar mejoras en la estabilidad y actividad biológica de los compuestos bioactivos provenientes de microalgas. Esta revisión persigue mostrar la importancia, mejoras y potencialidad en la elaboración de compuestos bioactivos de microalgas para productos nanoencapsulados con utilidad en la industria de alimentos.
\end{abstract}

Palabras claves: Alimento funcional. Bioproductos. Microalgas. Nanoencapsulación.

\section{Resumo}

As microalgas se apresentam como uma fonte potencial de compostos bioativos para utilização na indústria alimentícia, produzindo alimentos funcionais. O desenvolvimento e procura de alimentos funcionais, como método para melhorar e manter a saúde representa uma área substancialmente explorada nos últimos anos. Lipídeos, proteinas, polissacarídeos, compostos fenólicos, carotenóides, entre outros bioprodutos das microalgas podem ser utilizados como ingredientes para produtos funcionais, mas, a instabilidade química de muitos deles pode comprometer sua ação. A nanoencapsulação é uma ferramenta nanotecnológica capaz de proporcionar melhorias de estabilidade e atividade biológica dos compostos bioativos derivados de microalgas. Esta revisão procura mostrar a importância, melhoria e potencialidade na elaboração de compostos bioativos das microalgas para produtos nanoencapsulados com potencial para a indústria de alimentos.

Palavras chave: Alimentos funcionais. Bioprodutos. Microalgas. Nanoencapsulação. 


\section{Introducción}

Las microalgas, pertenecen a un grupo prometedor de microorganismos que merecen una atención considerable en vista de la amplia gama de procesos biotecnológicos en los cuales podrían estar involucrados. Estos microorganismos se caracterizan por poseer un metabolismo fotosintético y capacidad de desarrollar procesos autotróficos, heterotróficos y/o mixotrófico (Mitra et al., 2012). Las microalgas poseen un gran potencial ya que son capaces de convertir sustancias inorgánicas en compuestos orgánicos naturales que pueden ser utilizados como ingredientes funcionales (Gouveia et al., 2010). Muchos de estos compuestos son células enteras de microlgas (biomasa microalgal) o sus bioproductos metabólicos (compuestos bioactivos) generados durante su ciclo celular, y que se han utilizado como suplementos alimenticios o alimentos directos mostrando en ciertos casos resultados promisorios así como discretos (Zepka et al., 2010).

Existe una gran diversidad de microalgas y extractos que proporcionan elementos altamente nutricionales como proteínas, ácidos grasos esenciales y vitaminas. A su vez, representan una fuente en exploración de compuestos bioactivos que podrían ser útiles en aplicaciones farmacéuticas o nutracéuticas; así aparecen sustancias con efecto antimicrobiano, anti-inflamatorio, antialérgi$\mathrm{co}$, antioxidante, hipocolesterolémico, hipoglicemiante, inmunosupresor, antiagregante y vasoconstrictor, entre otros (Raposo et al., 2013).

La presencia de alimentos con actividad nutracéutica, producto de la adición de compuestos bioactivos, viene a ser un avance para el desarrollo de la alimentación. De esta forma, el suministro de estos alimentos, además de contribuir en la cadena nutritiva, aportaría elementos que beneficiarían la salud humana.

Sin embargo, muchos de estos compuestos bioactivos son inestables a cambios físicos o químicos por lo que al ser incorporados en alimentos atraviesan muchas barreras intrínsecas y extrínsecas que puede resultar en un aprovechamiento parcial o nulo por parte del consumidor. Por esto, la conservación de estos compuestos se convierte en un objetivo fundamental para conseguir un alimento funcional con éxito (Taksima et al., 2015)

La incorporación de la nanotecnología para el desarrollo de compuestos bioactivos aplicados en alimentos representa una metodología que ayudaría a la estabilidad de los mismos. La nanotecnología se muestra como una opción con gran potencial para la producción de alimentos suplementados con compuestos bioactivos de microalgas (Asmathunisha y Kathiresan, 2013).

La nanotecnología está invadiendo la industria de alimentos y se va estableciendo con un gran potencial. Entre las aplicaciones de la nanotecnología en la industria alimentaria se destacan el uso de nanopatículas y elementos nanoencapsulados lo que permite una absorción de nutrientes controlada, aumentos del sabor, alteración de color, seguridad alimentaria y aumento de vida útil en alimentos. Aunque la investigación en nanotecnología de alimentos está comenzando a impactar a entendidos, pudiendo causar una revolución en la industria de la alimentación, su aplicación es aun limitada. E s t e artículo busca mostrar la importancia de los compuestos bioactivos producidos por microalgas y que pueden ser aprovechados en alimentos con ayuda de procesos nanotecnológicos como la nanoencapsulación.

\section{Microalgas: compuestos bioactivos como fuente de alimentos funcionales}

El concepto de alimentos funcionales como sistema de protección y beneficios al consumidor nació por primera vez en Japón en 1980. A partir de allí surgieron muchos estudios y regulaciones por organismos como Foods for Specified Health Uses' (FOSHU, Japón), Food and Drug Administration (FDA, E.E.U.U) y la Functional Food Science in Europe (FUFOSE) (Arai, 1996; Diplock et al., 1999).

Un alimento funcional está constituido por elementos que además de proporcionar nutrientes, tienen un beneficio para una o más funciones del organismo humano, mejorando el estado de salud y reduciendo el riesgo a la enfermedad. Mucho de los compuestos metabólicos de microalgas, así como su biomasa tienen un efecto beneficioso, y pueden ser incorporados en alimentos (Tabla 1).

En el nivel de clasificación química, los compuestos con actividad biológica se pueden agrupar en ácidos grasos, proteínas, péptidos, aminoácidos, esteroles, pigmentos, vitaminas, alcaloides y otros compuestos no incluidos en estas clases.

En términos generales el contenido de proteínas en microalgas es elevado, la mayoría de las especies presentan cantidades por encima del 50\% de proteína en peso seco, recientemente se ha mostrado un gran interés con respecto a las proteínas de microalgas ya que poseen secuencia de aminácidos que forman péptidos bioactivos con gran potencial terapéutico en la salud humana, estos pueden proteger al organismo del deterioro por los radicales libres y especies reactivas de oxígeno (ROS) que pueden reaccionar con moléculas de DNA, proteínas y lípidos, llevando a muchos trastornos en la salud como inflamación, envejecimiento, diabetes, enfermedades cardiovasculares y cáncer (Kim y Kang, 2011).

Los péptidos se liberan de las proteínas parentales por la acción de una enzima (por lo general una proteasa); cuando son consumidos como péptidos puros o hidrolizados, ellos ejercen una función de modulación en la estructura y función de las enzimas metabólicas, implicadas en ciertas enfermedades, después de la absorción en el sistema circulatorio sanguíneo. Entre las actividades asociadas se incluye el efecto antioxidante, 
Tabla 1 - Compuestos bioactivos de microalgas y sus beneficios en salud humana

\begin{tabular}{|c|c|c|c|c|}
\hline Microalga & $\begin{array}{l}\text { Metabolito } \\
\text { biactivo }\end{array}$ & Actividad & $\begin{array}{c}\text { Aplicación en } \\
\text { salud }\end{array}$ & Referencia \\
\hline Chlorella protothecoides & Luteína & Antioxidante & $\begin{array}{l}\text { Protección de } \\
\text { regeneración macular }\end{array}$ & (Pulz y Gross, 2004) \\
\hline $\begin{array}{l}\text { Gambierdiscus toxicus, } \\
\text { Prorocentrum lima }\end{array}$ & Ácido okadaico & Antifúngico & Terapéutico & (Burja et al., 2001) \\
\hline Chlorella ellipsoidea & Violaxantin & Anticancerígeno & $\begin{array}{l}\text { Antiproliferativo } \\
\text { apoptótico }\end{array}$ & (Cha et al., 2008) \\
\hline Arthrospira/Spirulina & Ficocianina & $\begin{array}{l}\text { Antioxidante, } \\
\text { antiinflamatorio, } \\
\text { pigmento natural }\end{array}$ & $\begin{array}{l}\text { Suplemento } \\
\text { e ingrediente } \\
\text { alimentício mejora el } \\
\text { sistema inmune }\end{array}$ & (Pulz y Gross, 2004) \\
\hline Dunaliella salina & $\beta$-caroteno & $\begin{array}{l}\text { Aditivo alimentario, } \\
\text { antioxidante, pro- } \\
\text { vitamina A }\end{array}$ & $\begin{array}{l}\text { Suplemento } \\
\text { alimentario, mejora } \\
\text { el sistema inmune y } \\
\text { coagulación sanguínea }\end{array}$ & $\begin{array}{l}\text { (Guerin et al., 2003; } \\
\text { (Raposo et al., 2013) }\end{array}$ \\
\hline Haematococcus pluvialis & Astaxantina & $\begin{array}{c}\text { Pigmento natural, } \\
\text { antiinflamatorio, Aditivo } \\
\text { alimentario, antioxidante, } \\
\text { anticancerígeno y } \\
\text { antitumoral }\end{array}$ & $\begin{array}{c}\text { Suplemento } \\
\text { alimentario, } \\
\text { tratamiento del } \\
\text { síndrome del túnel } \\
\text { carpiano, inflamación } \\
\text { muscular, inductor de } \\
\text { apoptosis }\end{array}$ & $\begin{array}{c}\text { (Guerin et al., 2003; Pulz y } \\
\text { Gross, 2004) }\end{array}$ \\
\hline Chlorella vulgaris & $\begin{array}{c}\text { Péptido } \\
\text { VECYGPNRPQF }\end{array}$ & $\begin{array}{l}\text { Anticancerígeno y } \\
\text { antitumoral }\end{array}$ & $\begin{array}{l}\text { Antiproliferativo e } \\
\text { inductor de inhibición } \\
\text { post-G1 en células de } \\
\text { carcinoma gástrico }\end{array}$ & (Sheih et al., 2010) \\
\hline $\begin{array}{c}\text { Phaeodactylum tricornutum; } \\
\text { Porphyridium cruentum; } \\
\text { Crypthecodinium; Odontella; } \\
\text { Nannochloropsis; Pavlova lutheri; } \\
\text { Chorella sp. }\end{array}$ & $\begin{array}{l}\text { Ácidos grasos poli- } \\
\text { insaturados }\end{array}$ & $\begin{array}{c}\text { Nutracéutico, } \\
\text { antimicrobiano, } \\
\text { antiinflamatorio, } \\
\text { antiagregante, } \\
\text { vasoconstrictor } \\
\text { plaquetario, integridad } \\
\text { de tejidos; retraso del } \\
\text { envejecimiento }\end{array}$ & $\begin{array}{c}\text { Suplemento } \\
\text { alimenticio, combate } \\
\text { de infecciones y } \\
\text { dolencias }\end{array}$ & $\begin{array}{l}\text { (Pulz y Gross,2004; } \\
\text { Guedes, 2010) }\end{array}$ \\
\hline
\end{tabular}




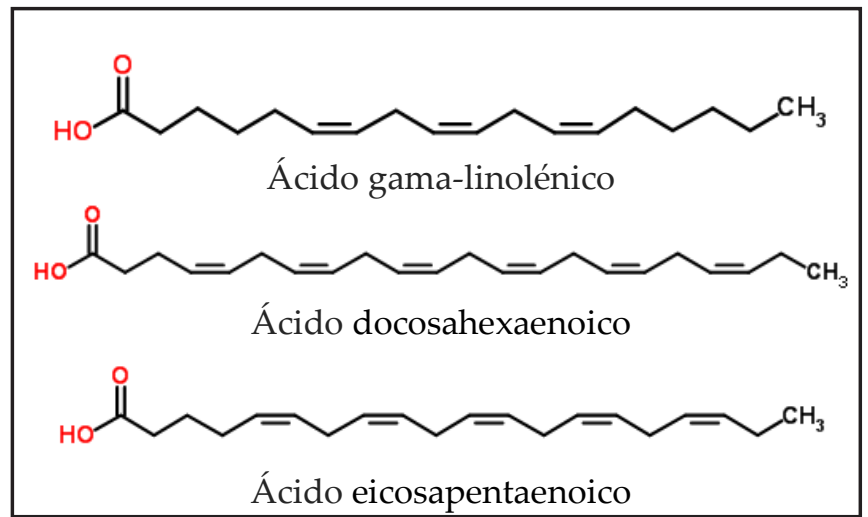

Figura 1- Ácidos grasos poliinsaturados presentes en microalgas

Disponible en http://www.chemspider.com/

lo que es muy importante para las industrias de alimentos. La oxidación en los alimentos afecta a los lípidos, proteínas e carbohidratos. La actividad antioxidante de péptidos bioactivos se debe principalmente a la presencia de aminoácidos hidrófobos, algunos aminoácidos aromáticos e histidina (Ngo et al., 2011). Varias líneas de estudio están tras la búsqueda de sustancias eficientes para la terapia contra la obesidad. Algunas proteínas de microalgas están asociadas al estímulo de la producción de la hormona colecistoquinina, un biomarcador asociado con la saciedad, que regula la supresión del apetito y se identifica como un elemento prometedor para la pérdida de peso, por lo tanto se han considerado en la formulación de alimentos funcionales contra el sobre peso (Ngoa et al., 2012).

El contenido y composición de los lípidos en microalgas varía entre especies y condiciones de habitad. Los principales lípidos polares en microalgas (mono y digalactosil diacilglicerol y fosfatidilglicerol) han descrito que poseen actividad anti-inflamatoria y anti-trombótica (Antonopoulou et al., 2005). Pero, los compuestos lipídicos más estudiados en las microalgas son los ácidos grasos poliinsaturados de cadena larga como los ácidos eicosapentaenoico (EPA, 20:5 $\omega$-3), docosahexaenoico (DHA, 22:6 $\omega$-3), araquidónico (ARA, 20:4 $\omega-6$ ), gamma -linolénico (GLA, 18:3 $\omega-6)$. La importancia de estos compuestos se basa en la incapacidad del humano para sintetizar algunos ácidos grasos, por lo que estos ácidos se denominan ácidos grasos esenciales. Estos ácidos grasos, especialmente la $\omega-3$ y $\omega-6$ son esenciales para la integridad de los tejidos en los que se incorporan. Los GLA son nutrientes esenciales para la síntesis de prostaglandinas, por el sistema inmunológico y otros procesos relacionados con la regeneración de tejidos. Los ácidos DHA y EPA están asociados a la reducción de problemas asociados con accidente cerebrovascular, artritis e hipertensión, y presentan actividad hipolipidémica significativa, mediante la reducción de triglicéridos y aumento de colesterol HDL. El DHA también participa en el desarrollo y el funcionamiento del sistema nervioso (Raposo et al., 2013).
Otros compuestos de lípidos bioactivos con propiedades interesantes son los esteroles. Ellos tienen un papel clave en la industria farmacéutica y nutracéutica, siendo los precursores de algunas moléculas bioactivas tales como vitaminas. Por otra parte, los fitosteroles han demostrado que reducen el colesterol-LDL (Francavilla et al., 2010).

Estas microalgas son potencialmente prometedores para la industria alimentaria como una valiosa fuente de ácidos grasos de cadena larga como una alternativa a los aceites de pescado, suministrando también esteroles (principalmente fitosterol), tocoferoles, pigmentos colorantes y otros nutracéuticos.

La Figura 1 muestra de forma representativa la estructura de tres ácidos grasos poliinsaturados producidos por microalgas.

En relación a los pigmentos, las cianobacterias particularmente sintetizan altos niveles de ficobiliproteínas, con porcentajes que alcanzan hasta $8 \%$ de su peso seco. Estos pigmentos se han utilizado como marcadores de fluorescencia no radiactivos cuando se une covalentemente a los anticuerpos, proteína A, biotina, lecitina y hormonas (Pérez-García et al 2011). Además de esta aplicación, las ficobiliproteínas tienen importante actividad antioxidante y anti-inflamatoria

Los carotenoides son otra clase importante de pigmentos encontrados de forma abundante en microalgas. Es bien conocido por la actividad pro-vitamina A de $\beta$-caroteno y su efecto sobre la visión y el sistema inmunológico. Por otra parte, la actividad antioxidante de los carotenoides está asociado con la prevención del cáncer, la arteriosclerosis, el envejecimiento y las enfermedades degenerativas (Rodrigues et al., 2015).

Como resultado de estas propiedades, muchos carotenoides han sido aprobados por organismos reguladores en varios países como colorantes naturales de alimentos y piensos. La astaxantina producida por Haematococcus pluvialis es la mayor fuente natural de este carotenoide y está relacionado con varios beneficios para la salud, como la protección contra la peroxidación lipídica, la degeneración macular relacionada a la edad, la reducción de la aterosclerosis, y el aumento de la respuesta inmune (Raposo et al., 2013).

La astaxantina se ha utilizado ampliamente en la alimentación de salmones, truchas y crustáceos como agente colorante. Por último, hay que considerar algunos ceto-carotenoides y carotenoides glicosilados encontrados exclusivamente en las células de microalgas, como mixoxantofila, equinenona y cantaxantina (Rodrigues et al., 2014). (Figura 2).

Las células de microalgas son ricas en vitamina $C, E$, tiamina, piridoxina, riboflavina, ácido nicotínico, biotina y tocoferol. Tales estructuras consolidadas tienen aplicaciones en el sistema inmune a través de la actividad antioxidante, la formación de células y coagulación de la sangre.

Las microalgas contienen grandes cantidades de po- 
Tabla 2 - Compuestos bioactivos de microalgas con efecto antimicrobiano

\begin{tabular}{|c|c|c|c|}
\hline $\begin{array}{l}\text { Cepa de microalga } \\
\text { y compuesto } \\
\text { bioactivo extraído }\end{array}$ & Estructura química & $\begin{array}{l}\text { Microorganismos } \\
\text { sensibles }\end{array}$ & Referencia \\
\hline $\begin{array}{l}\text { Fischerella ambigua } \\
\text { (Ambigol A) }\end{array}$ & & Bacillus subtilis & (Falch et al. 1993) \\
\hline $\begin{array}{l}\text { Nostoc commune } \\
\text { (Dipertenol) }\end{array}$ & & $\begin{array}{l}\text { Staphylococcus } \\
\text { epidermidis }\end{array}$ & (Jaki et al. 2000). \\
\hline $\begin{array}{l}\text { Óscillatoria redekei } \\
\text { (Ấcido coriólico) }\end{array}$ & & $\begin{array}{l}\text { Staphylococcus } \\
\quad \text { aureus }\end{array}$ & (Mundt et al. 2003) \\
\hline $\begin{array}{c}\text { Lyngbya sp } \\
\text { (Pahayokolide A) }\end{array}$ & & $\begin{array}{c}\text { Bacillus } \\
\text { megaterium, } B . \\
\text { subtilis }\end{array}$ & (Berry et al. 2004) \\
\hline $\begin{array}{l}\text { Scytonema sp. } \\
\text { (Scytoscalarol) }\end{array}$ & & $\begin{array}{l}\text { S. aureus, } \\
\text { B. anthracis, } M \text {. } \\
\text { tuberculosis }\end{array}$ & (Mo et al. 2009) \\
\hline $\begin{array}{l}\text { Fischerella ambígua } \\
\text { (fischambiguine } \mathrm{B} \text { ) }\end{array}$ & & $\begin{array}{c}\text { M. } \\
\text { tuberculosis (MIC } \\
2 \text { lM), S. aureus } \\
\text { (MIC 19.4 lM) and } \\
\text { M. smegmatis }\end{array}$ & (Mo et al. 2010) \\
\hline
\end{tabular}




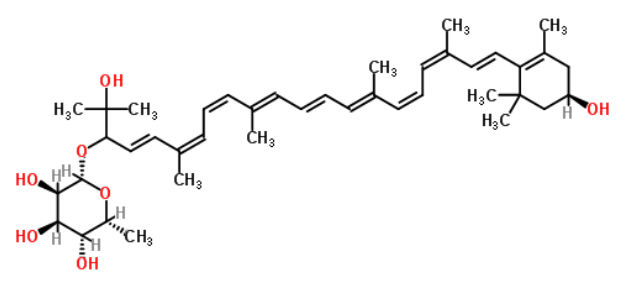

mixoxantofila

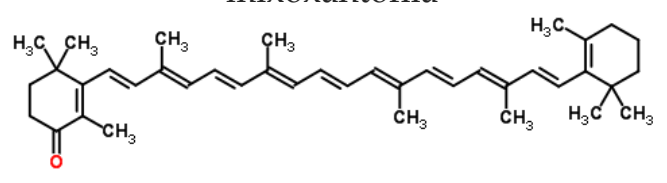

equinenona

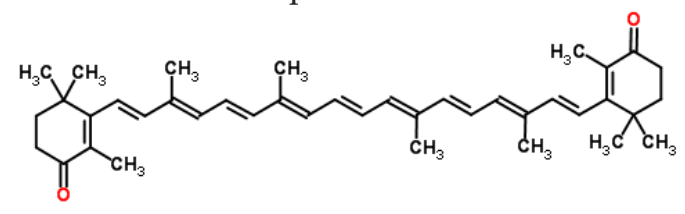

Cantaxantina

Figura 2- Carotenoides presentes en microalgas.

Disponible en http://www.chemspider.com/

lisacáridos, principalmente polisacáridos estructurales y de reserva. Dado que la mayoría de estos polisacáridos no son digeridos por los seres humanos, pueden ser consideradas como fibras dietéticas, con diferentes efectos fisiológicos asociados. Por ejemplo, la fibra insoluble (celulosa, hemicelulosa y lignina) promueve el movimiento de sustancias a través del sistema digestivo, mejorando así el efecto laxante, aumenta la sensación de saciedad, y mejora el crecimiento de la flora intestinal. Por otro lado, la fibra soluble (oligosacáridos, pectinas, $\beta$-glucanos, gomas y galactomanano) pueden ayudar con la hipocolesterolemia y regular la glucosa en sangre (Tosh y Yada, 2010).

La actividad antimicrobiana se ha probado tanto en extractos crudos como extractos hidrofílicos y lipofílicos de microalgas, mostrando que son una fuente rica en sustancias inhibidoras contra bacterias grampositivas y gramnegativas. Otra información importante que se determina en la evaluación de los extractos de microalgas para la actividad antibacteriana, es que la concentración mínima capaz de inhibir o matar a las bacterias está bastante por debajo de la recomendada por los controles de antibióticos estándar, además de actuar sobre bacterias multiresistentes (Mudimu et al., 2014). La tabla 2 muestra alguno de los compuestos de microalgas con efecto antimicrobiano.

\section{Importancia de la nanotecnología para el desarrollo de compuestos bioactivos de microalgas en la industria de alimentos}

En las últimas décadas, la evolución de una serie de nuevas disciplinas y tecnologías científicas han revolucionado el sector de la alimentación. Si bien la aplicación de la nanotecnología en el sector alimentario se presenta como un método emergente, se prevé que crezca rápidamente en los próximos años. La nanotecnología es una plataforma tecnológica donde nuevos nanomateriales ofrecen muchas oportunidades para la industria alimentaria, como coloración más potente para alimentos, aromas, aditivos nutracéuticos e ingredientes antimicrobianos como suplemento alimenticio. De esta tecnología surge la nanoencapsulación, donde se empaquetan sustancias con ingrediente activo en una cápsula polimérica de tamaño menor a 100 nm. Aquí se hace uso de técnicas como nanoencapsulación, nanoemulsificación y nanoestructuración, proporcionando mayor funcionalidad del producto final que incluye la liberación controlada del compuesto activo (Chellaram et al., 2014).

Mucho de los compuestos bioactivos de microalgas referidos son susceptibles a condiciones intrínsecas o extrínsecas por lo que su actividad biológica se ve afectada. La inestabilidad se muestra en muchas moléculas, por ejemplo el carotenoide extraído de Chlorella vulgaris y Haematococcus pluvialis cuando fueron incorporadas en la dieta del pez dorado (Sparus aurata) mostraron una estabilidad muy corta en relación a las moléculas encapsuladas donde se pueden mantener en periodos largos sin cuidado de las condiciones de almacenamiento (Gouveia y Empis et al., 2003).

De esta forma, la nanoencapsulación proporciona protección de los compuestos bioactivos alcanzando una producción mejorada de alimentos funcionales, ya que la hace más estable y específica para una liberación controlada logrando una absorción eficiente de la sustancia activa lo que ha sido siempre un gran desafío.

Las diferentes técnicas desarrolladas para la producción de nanocápsulas se han reportado, junto con ejemplos de su aplicación. Así, productos como NuMega Driphorm incorporan nanocápsulas de omega-3 como aditivo alimenticio en preparados lácticos para lactantes, niños en crecimiento, adultos, además en otras aplicaciones alimentarias, que se puede evidenciar en el enlace: http://www.clovercorp.com.au/en/discover-our-products/dha-powders/

Algunos procesos de nanoencapsulación de compuestos bioactivos con actividad nutracéutica se han patentado. La capacidad de protección que ofrece una nanocápsula permite que el compuesto biactivo se preserve a su paso por vía digestiva, tanto el compuesto que forma la nanocápsula como el compuesto bioactivo son absorbidos, este último atinge al órgano en el cual hará su efecto, mientras que el otro será excretado (Livney, 2012).

En base a esto, la síntesis de nanocápsulas de origen microalgal representaría una oportunidad para suplementar alimentos con doble acción nutracéutica. Así la elaboración de nanocápsulas a partir de polisacáridos 


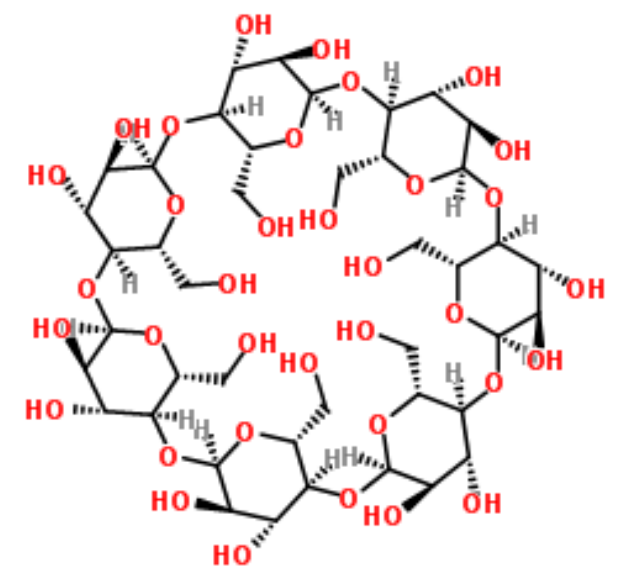

$\beta$-Ciclodextrina

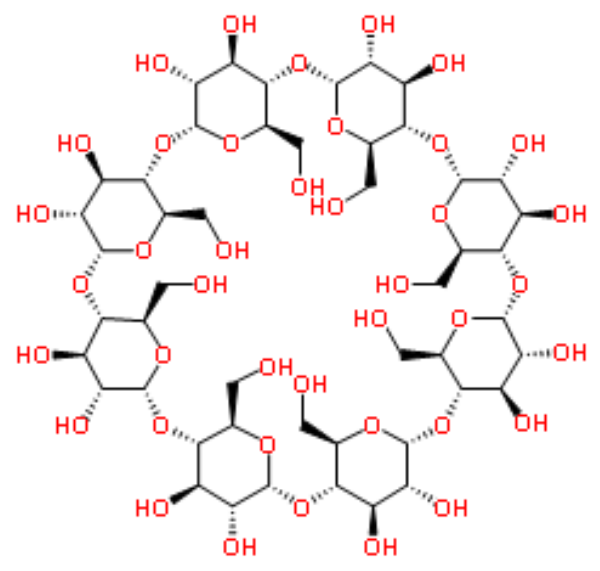

$\gamma$-Ciclodextrina

Figura 3- Ciclodextrinas utilizada para nanoencapsulación de compuestos activos.

Disponible en http://www.chemspider.com/

sulfatados, con demostrado efecto antiviral y antitumoral, provenientes de Porphyridium, Rhodella y la cianobacteria Arthrospira (Gardeva et al., 2009); que recubran moléculas de $\beta$-caroteno aisladas de Dunaliella salina con conocido efecto antioxidante y anticancerígeno (Raposo et al., 2013), podrían ser utilizados en pacientes con leucemias de células blancas. En este tipo de pacientes la leucopenia conlleva a numerosas infecciones, así la acción bioactiva antitumoral actuaría sobre leucocitos inhibiendo su proliferación mientras que la acción antiviral ayudaría a combatir infecciones por gérmenes.

Las ciclodextrinas se han utilizado con éxito para encapsular una amplia gama de ingredientes funcionales solubles mediante la formación de complejos de inclusión molecular. Esta molécula se presenta como una forma de almidón modificado con una estructura de anillo formada por unidades de glucosa. Los más comúnmente utilizados para nanacápsulas son la beta y el gamma ciclodextrina los cuales poseen 7 y 8 anillos respectivamente (Figura 3).

Ellos tienen un pequeño diámetro exterior de menos de $2 \mathrm{~nm}$ y una superficie externa polar lo que les permite disolverse en agua. Su núcleo interior forma una cavidad no polar que puede contener pequeñas moléculas lipofílicas, como por ejemplo astaxantina proveniente de Haematococcus pluvialis, esteroles de Chaetoceros y Skeletonema, vitaminas e Isochrysis galbana, ácidos grasos poliinsaturados y ficobiliproteína de Arthrospira platensis. Las nanocápsulas de ciclodextrinas permiten una mejor propiedad de disolución de los compuestos bioactivos en su interior que poseen escasa solubilidad en agua o que poseen baja estabilidad.

Las ciclodextrinas se han utilizado durante muchos años para estabilizar sabores. Se ha utilizado principalmente en la industria cervecera, para mejorar la estabilidad de los productos que contienen compuestos de sabor amargo, tales como flavonoles (quercetina y miricetina).
Con igual aprovechamiento el uso de ciclodextrinas está siendo adoptadas por las industrias de alimentos, bebidas y agua; de esta forma se busca enmascarar sabores como el ginseng y té verde agregados en bebidas (Vance et al., 2015; Lu et al., 2013)

Las proteínas también tienen propiedades anfolíticas y pueden ser utilizados de una manera análoga a las formas de micelas estables. Las micelas de caseína se están investigando por su capacidad de llevar compuestos bioactivos que pueden ser utilizados en bebidas claras y bebidas deportivas (Domingos et al., 2009). Las micelas estables incorporados con lípidos, cuya distribución de tamaño de partícula es aproximadamente $50 \mathrm{~nm}$, se pueden formar usando extracto vegetal de Quillay (Quillaja saponaria) y ha sido recientemente desarrollado para su uso en bebidas (Liao et al., 2014).

\section{Nanocápsulas para nanoencapsulación}

Como se mencionó, la nanoencapsulación persigue preservar la estabilidad del compuesto bioactivo. De igual forma, busca mantener el control en la liberación del compuesto bioactivo para que estos alcancen al órgano específico al cual está destinado, conservando la concentración que el fabricante del alimento funcional espera aportar al consumidor. Grupos de investigación han desarrollado nuevas tecnologías y patentes para mejorar la acción de los compuestos nanoencapsulados (Alonso et al., 2014).

Diversas investigaciones examinan la encapsulación y liberación controlada de compuestos activos para uso en alimentos utilizando un enfoque nanotecnológico. Así, es una molécula que puede ser bifuncional como el anhídrido octenil succinato $\varepsilon$-poli(lisina) puede ser utilizada ya sea como agente tensioactivo o emulsionante en la encapsulación de compuestos bioactivos o como 
agentes antimicrobianos (Yu et al., 2009)

Los sistemas de nanoencapsulación basado en lípidos optimizan el rendimiento de compuestos antioxidantes mediante la mejora de su solubilidad y biodisponibilidad, en vitro y en vivo, además preserva las interacciones no deseadas con otros componentes de alimentos. Entre estos sistemas los nanoliposomas, arqueosomas y nanocochleates. Su tamaño único y las propiedades hidrofílicas/hidrofóbicas les permiten atrapar, entregar, y liberar materiales solubles en agua y lípidos (Rao y McClements, 2012). Los Nanoliposomas se presentan como una interesante oportunidad para la industria de alimentos en áreas tales como la encapsulación y liberación controlada de productos alimenticios, así como la biodisponibilidad mejorada, estabilidad y vida útil de los ingredientes sensibles. Ya se ha reportado la aplicación de nanoliposomas en el campo de alimentos como instrumento de conducción de nutrientes, nutracéuticos, enzimas, aditivos alimenticios y antimicrobianos (Mozafari et al., 2008).

Los coloidosomas, que son una estructura autoensamblada de partículas coloidales que pueden formar estructuras huecas capaces de transportar compuestos bioactivos en su interior y depositarlo de manera controlada en un blanco determinado. Otro sistema de administración para nanocápsulas muy estable y preciso está representado por los cochleates, este es un fosfolípido divalente compuesto por materiales de origen natural, el cual fue desarrollado y patentadas por BioDelivery Sciences International Inc., Newark, U.S.A. (Ranjan et al., 2014).

Otro de los sistemas (nanocochleates) tiene una estructura de capas múltiples que consiste en una lámina de bicapa lípido-sólido continuo enrollado en forma de espiral con poco o ningún espacio acuoso interno. La estructura nanocochleate proporciona protección y estabilidad durante la conducción de compuestos bioactivos con características hidrofílicas y lipofílicas lo que aumenta el valor nutritivo de los alimentos procesados (Bhosale et al., 2013).

Los sistemas de nanoencapsulación basado en polímeros, son moléculas compuestas de regiones típicamente opuestas (hidrofílicas e hidrofóbicas). Algunos de estos biopolímeros utilizados como agentes nanoencapsulantes son las proteínas (albumina, gelatina, colágeno, $\alpha$-lactoalbumina y los polisacáridos (alginato, chitosan). Las proteínas son de mayor interés en vista que ellas son relativamente fácil de preparar y pueden formar complejos con polisacáridos, lípidos, biopolímeros y con una gran variedad de nutrientes (Chen et al., 2006).

La nanoencapsulación puede proporcionar un ahorro significativo para los formuladores, ya que puede reducir la cantidad de ingredientes activos necesarios. Sin embargo, es fundamental la comprensión de las interacciones a nivel molecular que se dan entre los polímeros y el compuesto activo, para así evitar impactar sobre sus propiedades funcionales y garantizar un uso adecuado en la industria alimentaria

\section{Métodos para preparación de nanocáp- sulas}

Existen varios métodos para la preparación de nanocápsulas poliméricas, los cuales pueden ser, de una forma general, clasificados en los métodos basados en la polimerización in situ de monómeros dispersos (cianoacrilato de alquilo) (Lambert et al., 2000), o en la proliferación de polímeros preformados como poli-ácido láctico, poli-ácido láctico-co-ácido glicólico, poli-ع-caprolactona y copolímeros de ácido metacrílico (Marchais et al., 1998).

La elección de la técnica adecuada para cada sustancia es primordial para lograr los resultados esperados. Esta elección se debe hacer en función tanto de las propiedades de la sustancia a encapsular como del sistema de liberación deseado para cada aplicación. Entre las técnicas para el desarrollo y aplicación de la encapsulación, según Assis et al., (2012) tenemos:

La coacervación compleja. En este método, dos polímeros solubles en agua y con cargas diferentes son mezclados para la formación de un complejo entre polímeros que conduce a la deposición del recubrimiento, denominado coacervato, sobre las gotas de una emulsión de la sustancia activa. La formación del coacervato está controlada por la adición de una sal, el pH, temperatura o por la dilución del medio.

Polimerización Interfacial: Este método es adecuado para la encapsulación de agentes activos en estado líquido. La base de esta técnica consiste en la reacción de polimerización de monómeros en la interfase de dos líquidos inmiscibles dando lugar a la pared o membrana de la microcápsula. La reacción de polimerización está controlada por difusión, y el tamaño de las microcápsulas lo determina las propiedades de la emulsión inicial.

Polimerización en miniemulsión: Las miniemulsiones son dispersiones estabilizadas que pueden alcanzar tamaños de aproximadamente 50 - $100 \mathrm{~nm}$, son obtenidas mediante la utilización de ultrasonidos, cuya característica principal es la elevada estabilidad de la emulsión. Este método permite la obtención de nanocápsulas mediante procesos de polimerización interfacial como el descrito anteriormente.

Preparación de coloidosomas: Estos son cápsulas cuya pared está formada por la coagulación, agregación o fusión de partículas, dando lugar a recubrimientos porosos. El grado de permeabilidad del recubrimiento puede controlarse durante el proceso de formación del coloidosoma mediante la temperatura y tiempo de reacción. De esta forma se obtienen microcápsulas con 
un perfil de liberación gradual y sostenido, en la que la sustancia activa se libera a lo largo del tiempo mediante difusión a través de la cubierta porosa.

Evaporación del disolvente o deposición polimérica: Este método está basado en el fenómeno de separación de fases y se utiliza habitualmente en emulsiones. La fase orgánica consiste en una mezcla del polímero que más tarde formará la cubierta, un disolvente volátil y otro no volátil. A medida que el disolvente volátil se evapora, el polímero precipita en la superficie de la sustancia activa atrapándola en su interior y dando lugar a las cápsulas.

De esta forma, en función de la aplicación, tipo de compuesto, material polimerico, entre otros se podrá seleccionar el mecanismo más adecuado para la liberación del material encapsulado.

\section{Mejoras para la producción y acción de compuestos bioactivos de microalgas}

\subsection{Aspectos del cultivo}

Como ya fue mencionado, las microalgas tienen un gran potencial para la producción de compuestos bioactivos a ser utilizados como nutracéuticos en la industria de alimentos y farmacéutica en vista de los amplios beneficios mostrados.

Aunque cientos de estos metabolitos se han identificado en cultivos de microorganismos fotosintéticos, la inducción de la síntesis en la mayoría de los casos es conocida, la separación y recuperación de los compuestos tienen que ser optimizados y por lo tanto la inclusión en la formulación de productos comerciales aún requiere de investigación y desarrollo.

A pesar de estas limitaciones, la producción de compuestos bioactivos de microalgas depende en gran medida de la cepa escogida junto con las condiciones de cultivo y utilización de fotobiorreactores adecuados (Ramírez-Mérida et al., 2015). Por lo tanto, la producción de metabolitos puede ser optimizado teniendo en cuenta diferentes enfoques para el aislamiento y purificación de la molécula diana y la confirmación de la actividad biológica.

\subsection{Nanotecnología}

Muchas de las grandes empresas de alimento a nivel mundial están invirtiendo en nanotecnología y están en camino a la comercialización de nuevos productos, esto con el fin de ofrecer productos alimenticios con una mejor estructura y condición organoléptica, así como proveer sustancias bioactivas que puedan proporcionar beneficios en la salud del consumidor. En esto, la nanoencapsulación ofrece una mayor biodisponibilidad, solubilidad mejorada y mayor potencia en comparación con sustancias no encapsuladas o microencapsulado. De igual manera, la potencia de los aditivos de nanopartículas también puede reducir las cantidades de los aditivos necesarios, y también su mayor reactividad química (Vance et al., 2015).

Sin embargo, una de los obstáculos encontrados son las regulaciones. La tecnología empleada es un tema fundamental y es el factor más influyente hacia la mayor parte de los nuevos alimentos. Hay una discusión en el momento en Europa sobre qué tecnologías deben y qué tecnologías no deben ser incluidos en el reglamento sobre nuevos alimentos, como es el caso del uso de nanotecnología en el tratamiento y la formulación de compuestos bioactivos de microalgas (Enzing et al., 2014).

\section{Consideraciones finales}

La incorporación de microalgas en sistemas productivos de industrias de alimentos proporciona algunas ventajas ya que el carácter natural, fácil cultivo, su rápido crecimiento (para muchas de las especies) y la posibilidad de controlar la producción de algunos compuestos bioactivos mediante la manipulación de las condiciones de cultivo, hace que las microalgas puedan ser consideradas como auténticos reactores naturales, en algunos casos, una buena alternativa a la síntesis química de ciertos compuestos.

La nanoencapsulación de estos compuestos en alimentos y piensos no solo proporciona estabilidad a compuestos bioactivos en el tiempo, sino que además proporciona oportunidades para la innovación biotecnológica en esta área. Cada día se tiene más acceso a las herramientas tecnológicas para que podamos aprender de los procesos de nano escala que operan en nuestro entorno cotidiano, y en los próximos años podremos conocer muchos de los principios biológicos de la nanotecnología, así como incorporación sin restricciones de estos productos en anaqueles comerciales.

\section{Referencias}

Alonso, F.M.J., López, D.T., Rivera, R.G., Oyarzún A.F.A., Lollo, G., Lázaro, G.T., Garcia, M.F. (2014). Nanocapsules with a polymer shell. US Patent 20140023703 A1, Jan. 23.

Antonopoulou, S., Nomikos, T., Oikonomou, A., Kyriacou, A., Andriotis, M., Fragopoulou, E., Pantazidou, A. (2005). Characterization of bioactive glycolipids from Scytonema julianum (cyanobacteria). Comparative Biochemistry and Physiology Part B: Biochemistry and Molecular Biology, 140(2), 219-31.

Arai,S.(1996).Studies on functional foodsinJapan-state of the art. Bioscience, Biotechnology, and Biochemistry, 60, 9-15. 
Asmathunisha, N., Kathiresan, K. (2013). A review on biosynthesis of nanoparticles by marine organisms. Colloids and Surfaces B: Biointerfaces, 103, 283-287.

Assis, L.M., Zavareze, E.R. Prentice-Hernández, C., Souza-Soares, L.A. (2012). Characteristics of nanoparticles and their potential applications in foods. Brazilian Journal of Food Technology, 15(2), 99-109.

Berry, J.P., Gantar, M., Gawley, R.E., Wang, M., Rein, K.S. (2004). Pharmacology and toxicology of pahayokolide A, a bioactive metabolite from a freshwater species of Lyngbya isolated from the Florida Everglades. Comparative Biochemistry and Physiology: Toxicology \& Pharmacology, 139, 231-238.

Bhosale, R.R., Ghodake, P.P., Mane, A.N., Ghadge, A.A. (2013). Nanocochleates: A novel carrier for drug transfer. Journal of Scientific and Innovative Research, 2(5), 964-969.

Burja, A.M., Banaigs, B., Abou-Mansour, E., Burgess, J.G., Wright, P.C. (2001). Marine cyanobacteria - aprolific source of natural products. Tetrahedron, 57(46), 9347-77.

Cha, K.H., Koo, S.Y., Lee, D.U. (2008). Antiproliferative effects of carotenoids extracted from Chlorella ellipsoidea and Chlorella vulgaris on human colon cancer cells. Journal of Agricultural and Food Chemistry, 56, 10521-10526.

Chellaram, C. Murugaboopathi, G., John A.A., Sivakumar, R., Ganesan, S., Krithika, S., Priya G. (2014). Significance of Nanotechnology in Food Industry. APCBEE Procedia, 8, 109-113.

Chen, L.Y., Remondetto, G.E., Subirade, M. (2006). Food protein based materials as nutraceutical delivery systems. Trends in Food Science Technology, 17, 272-283.

Diplock, A.T., Aggett, P.J., Ashwell, M., Bornet, F., Fern, E.B., Roberfroid, M.B. (1999). Scientific Concepts of Functional Foods in Europe: Consensus Document. British Journal of Nutrition, 81(1), S1-S27.

Domingos, R.F., Baalousha, M.A., Ju-Nam, Y.M., Reid, M., Tufenkji, N., Lead, J.R., Leppard, G.G., Wilkinson, K.J. (2009). Characterizing manufactured nanoparticles in the environment: multimethod determination of particle sizes. Environmental Science and Technology, 43, 7277-7284.

Enzing, C., Ploeg, M., Barbosa, M., \& Sijtsma, L. Microalgae based products for the food and feed sector: an outlook for Europe. In Vigani, M., Parisi, C., Rodríguez Cerezo, E. (Eds.), JRC scientific and policy reports. Luxembourg: Publications Office of the European Union; 2014. p. 49-60.

Falch, B.S., Konig, G.M., Wright, A.D., Sticher, O., Ruegger, H., Bernardinelli, G. (1993). Ambigol A and
B: new biologically active polychlorinated aromatic compounds from the terrestrial blue-green alga Fischerella ambigua. Journal of Organic Chemistry, 58, 6570-6575.

Francavilla, M., Trotta, P., Luque, R. (2010). Phytosterols from Dunaliella tertiolecta and Dunaliella salina: A potentially novel industrial application. Bioresource Technology, 101, 4144-4150.

Gardeva, E., Toshkova, R., Minkova, K., Gigova, L. (2009). Cancer protective action of polysaccharide derived from microalga Porphyridium cruentum-a biological background. Biotechnology \& Biotechnological Equipment, 23(1), 783-787.

Gouveia, L., Empis, J. (2003). Relative stabilities of microalgal carotenoids in microalgal extracts, biomass and fish feed: effect of storage conditions. Innovative Food Science and Emerging Technologies, 4, 227-233.

Gouveia, L., Marques, A.E., Sousa, J.M., Moura, P., Bandara, N.M. (2010). Microalgae-source of natural bioactive molecules as functional ingredients. Food Science \& Technology Bulletin: Functional Foods, 7, 21-37.

Guedes, A.C.A. (2010). Production, extraction and characterization of selected metabolites from microalgae and cyanobacteria. PhD ThesisPorto, Portugal: Escola Superior de Biotecnologia, Universidade Católica Portuguesa.

Guerin, M., Huntley, M.E., Olaizola, M. (2003). Haematococcus astaxanthin: applications for human health and nutrition. Trends in Biotechnology, 21(5), 210-216.

Jaki, B., Heilmann, J., Sticher, O. (2000). New antibacterial metabolites from the cyanobacterium Nostoc commune (EAWAG 122b). Journal of Natural Products, 63, 1283-1285.

Kim, S.K, Kang, K.H. (2011). Medicinal effects of peptides from marine microalgae. Advances in Food and Nutrition Research, 64, 313-323.

Lambert, G., Fattal, E., Pinto-Alphandary, H., Gulik,,A., Couvreur, P. (2000). Polyisobutyl cyanoacrylate Nanocapsules Containing an Aqueous Core as a Novel Colloidal Carrier for the Delivery of Oligonucleotides. Pharmaceutical Research, 17, 707-714.

Liao, H.G., Zherebetskyy, D., Xin, H., Czarnik, C., Ercius, P., Elmlund, H., Pan, M., Wang, LW., Zheng, H. (2014). Facet development during platinum nanocube growth. Science, 345(6199), 916-919.

Livney, Y.D. (2012). Protein-polysaccharide conjugates and use for encapsulating nutraceuticals for clear beverage applications. US Patent 20120288533 A1, Nov. 15. 
Lu, J., Viwat, S., Lu, S. (2013). Nano-flavor and preparation method thereof. WO Patent 2013004003, Jan. 25.

Marchais, H., Benali, S., Irache, J.M., Tharassebloch, C., Lafont, O., Orecchioni, A.M. (1998) Entrapment efficiency and initial release of phenylbutazone from nanocapsules prepared from different polyesters. Drug Development and Industrial Pharmacy, 24, 883-888.

Mitra, D., van Leeuwen, J.H, Lamsal, B. (2012). Heterotrophic/ mixotrophic cultivation of oleaginous Chlorella vulgaris on industrial co-products. Algal Research, 1(1), 40-48.

Mo, S., Krunic, A., Pegan, S.D., Franzblau, S.G., Orjala, J. (2009). An antimicrobial guanidine-bearing sesterterpene from the cultured cyanobacterium Scytonema sp. Journal of Natural Products, 72, 2043-2045.

Mo, S., Krunic, A., Santasiero, B.D., Franzblau, S.G., Orjala J. (2010). Hapalindole-related alkaloids from the cultured cyanobacterium Fischerella ambigua. Phytochemistry, 71, 2116-2123.

Mozafari, M.R, Johnson, C., Hatziantoniou, S., Demetzos, C. (2008). Nanoliposomes and their applications in food nanotechnology. Journal of Liposome Research, 18(4), 309-327.

Mudimu, O., Rybalka, N., Bauersachs, T., Born, J., Friedl, T., Schulz, R. (2014). Biotechnological screening of microalgal and cyanobacterial strains for biogas production and antibacterial and antifungal effects. Metabolites, 4, 373-393.

Mundt, S., Kreitlow, S., Jansen, R. (2003). Fatty acids with antibacterial activity from the cyanobacterium Oscillatoria redekei HUB 051. Journal of Applied Phycology, 15, 263-267.

Ngo, D.H., Wijesekara, I., Vo, T.S., Ta, Q.V., Kim, S.K. (2011). Marine food-derived functional ingredients as potential antioxidants in the food industry: an overview. Food Research International, 44, 523-529.

Ngoa, D.H., Voa, T.S., Ngob, D.N., Wijesekarac, I., Kima, S.K. (2012). Biological activities and potential health benefits of bioactive peptides derived from marine organisms. International Journal of Biological Macromolecules, 51, 378- 383.

Perez-Garcia, O., Escalante, F.M.E., de-Bashan, L.E., Bashan, Y. (2011). Heterotrophic cultures of microalgae: metabolism and potential products. Water Research, 45(1), 11-36.

Pulz, O., Gross, W. (2004). Valuable products from biotechnology of microalgae. Applied Microbiology and Biotechnology, (65), 635-48.
E. (2015). Why does the Photobioreactors Fail? Journal of Bioprocessing \& Biotechniques, 5, 7.

Ranjan, S., Dasgupta, N., Chakraborty, A.R., Samuel, S.M., Chidambaram, R., Rishi, S., Kumar, A. (2014). Nanoscience and nanotechnologies in food industries: Opportunities and research trends. Journal of Nanoparticle Research, 16, 2464.

Rao, J., McClements, D.J. (2012). Food-grade microemulsions and nanoemulsions: Role of oil phase composition on formation and stability. Food Hydrocolloids, 29, 326-334.

Raposo, M.F.J., Morais, R.M.S.C., Morais, A.M.M.B. (2013). Health applications of bioactive compounds from marine microalgae. Life Sciences, 93, 479-486.

Rodrigues, D.B., Flores, É.M.M., Barin, J.S., Mercadante, A.Z., Jacob-Lopes, E., Zepka L.Q. (2014). Production of carotenoids from microalgae cultivated using agroindustrial wastes. Food Research International, 65(B), 144-148.

Rodrigues, D.B., Menezes, C.R., Mercadante, A.Z., Jacob-Lopes, E., Zepka, L.Q. (2015). Bioactive pigments from microalgae Phormidium autumnale. Food Research International, In Press, Corrected Proof.

Sheih, I.C., Fang, T., Wu, T.K., Lin, P.H. (2010). Anticancer and antioxidant activities of the peptide fraction from algae protein waste. Journal of Agricultural and Food Chemistry, 58 (2), 1202-1207.

Taksima, T., Limpawattana, M., Klaypradit, W. (2015). Astaxanthin encapsulated in beads using ultrasonic atomizer and application in yogurt as evaluated by consumer sensory profile. LWT - Food Science and Technology, 62, 431-437.

Tosh, S.M., Yada, S. (2010). Dietary fibres in pulse seeds and fractions: Characterization, functional attributes, and applications. Food Research International, 43, 450-460.

Vance, M. E., Kuiken, T., Vejerano, E. P., McGinnis, S. P., Hochella, M. F., Jr., Rejeski, D., Hull, M. S. (2015). Nanotechnology in the real world: Redeveloping the nanomaterial consumer products inventory. Beilstein Journal of Nanotechnology, 6, 1769-1780.

Yu, H., Huang, Y., Huang, Q. Synthesis and characterization of novel antimicrobial emulsifiers from $\varepsilon$-polylysine. Journal of Agricultural and Food Chemistry, 58(2), 1290-1295

Zepka, L.Q., Jacob-Lopes, E., Goldbeck, R., Souza-Soares, L.A., Queiroz, M.I. (2010). Nutritional evaluation of single-cell protein produced by Aphanothece microscopica Nägeli. Bioresource Technology, 101, 7107-7111.

Ramírez-Mérida, L.G., Zepka, L.Q., Jacob-Lopes, 